Revista de Psicología de la PUCP. Vol. XIV. No 2. 1996

\title{
A PROPÓSITO DE LAS RELACIONES DE GÉNERO: ¿IDENTIDAD GENÉRICA O ESTILOS DE INTERACCIÓN DE GÉNEROS?
}

\author{
Aldo Bazán Ramírez' \\ Universidad Nacional Autónoma de México
}

En el presente trabajo se discuten algunos supuestos del determinismo social en la construcción de roles de género e identidades genéricas estereotipadas y de desigualdad en las relaciones intergéneros. Se propone que los estilos de interacción de los géneros se constituyen como formas o disposiciones relativamente invariantes en relación a contexros particulares de interacción social. No es suficiente afirmar que los factores de socialización y las primeras relaciones en grupo social, generan una identidad genérica estereotipada o de segregación de géneros, sino que es necesario también explicitar el cómo se estructuran preferencias, creencias y estilos de interacción de acuerdo a tales factores $\mathrm{y}$, cómo estas tendencias interactivas se constituyen a partir de situaciones de interacción social y como proceso de desarrollo. Palabras claves: Género, identidad, roles, estilos de interacción, tendencias interactivas.

Regarding gender relations: Gender identity or gender interaction styles?

This study discusses some assumptions from the social determinism in the construction of gender roles, gender-typed identities, and gender relations inequities. It is proposed that gender styles of interaction are relatively invariant forms or dispositions related to specific contexts of social interaction. It is not enough to say that socialization factors such as the family, school, mass media, and the initial social group relations generate a typed gender identity or gender seggregation, but it is also necessary to make explicit how preferences, beliefs and interaction styles are structured according to those factors and how these interactive tendencies are built upon social interaction situations as part of a developmental process.

Key words: Gender, identity, roles, interaction styles, interactive tendencies.

1 Graduado de la Facultad de Psicología en la Universidad Nacional Federico Villarreal en Lima, Perú; Egresado de la Maestría de Psicología opción en Metodología y Teoría de la Investigación Conductual en la Universidad Nacional Autónoma de México.

Dirección: Rancho la Esmeralda N. 62, 8-"B”, Dpto. 101. Col. Prados del rosario, Azcapozalco. C.P. 02410. Distrito Federal, México. Correo electrónico: aldo@servidor.unam.mx 



\section{Introducción}

La categoría género se ha asumido, fundamentalmente, como conceprualización de las relaciones que se establecen entre los individuos para explicar que la desigualdad e inequidad en las relaciones entre los individuos no son determinadas por, o no se establecen específicamente debido a las diferencias biológicas, económicas o generacionales, sino, más bien, para referirse al cómo estas variables influyen en el establecimiento de relaciones de desigualdad, de segregación y de sometimiento de unos individuos sobre otros. A juicio del autor del presente trabajo, el problema en el análisis y explicación de las relaciones de género radica en limitar la explicación del fenómeno psicológico a partir de variables sociales, como por ejemplo, el señalar que los roles y estilos de interacción se deben a variables culturales y modelos sociales los cuales determinan el aprendizaje e interiorización de estereotipos y roles del ser varón o el ser mujer. Aún cuando los supuestos culturalistas significan la superación de explicaciones del determinismo biológico que fundamentaban que estas diferencias se debían a las diferencias biológicas existentes entre los seres humanos, han orientado a una sobrevaloración de lo social en el desarrollo de estilos de interacción de géneros.

En este trabajo se considera que el género es implicancia de la Psicología en tanto refiere estilos de actuar y tendencias de interacción de los individuos frente a otras personas, objetos y hechos que forman parte de un contexto de interacción. Los estilos y tendencias de interacción se constituyen bajo criterios de mutua afectación y correspondencia funcional entre esos individuos y el ambiente tanto físico como normativo, lo cual es posibilitado por lo biológico como condición necesaria en el proceso de desarrollo psicológico. Por otra parte, las distintas situaciones de interacción, como la familia, la guardería infantil, la escuela primaria, los 
grupos de amigos, los medios de comunicación, entre otros, median la interacción de los individuos y, además, constituyen la situaciones que posibilitan tales interacciones. Así pues, la segregación o discriminación de algunos individuos por otros individuos por pertenecer a otra clase social, raza, sexo o generación, está en función a la historia interactiva de cada uno de esos individuos y a los criterios de logro que se asumen que deben cumplir los miembros de cada grupo de acuerdo a variables como raza, sexo, edad, etnia y, a las creencias y valoraciones que asumen estos sujetos frente a individuos que pertenecen a otros grupos o hacia personas del sexo opuesto.

De esta manera, las relaciones de género implican modos o estilos en que los individuos se relacionan en situaciones concretas de interacción. Es decir, los estilos de interacción de géneros permiten describir las tendencias interactivas de los individuos tanto al interior de cada género, como también en la relación intergéneros, de acuerdo a variables como sexo, edad, raza, clase socioeconómica, entre otras. Estos estilos de interacción no son causados por lo social per se, sino que se constituyen como proceso de historicidad específica y del modo de organización particular de los eventos con los que cada individuo entra en contacto funcional. Este proceso está vinculado muy estrechamente al desarrollo de actitudes y competencias conductuales en esas situaciones particulares de interacción social.

\section{Género y desarrollo de lo psicológico}

El género, como categoría de análisis, hace referencia al modo en que se constituye lo psicológico en contextos de interacción en situaciones establecidas convencionalmente, y no como muchos creen, que implica "mujerología" o una especie de estudio de la condición de la Mujer solamente. Autoras como Rubin (1984), Lamas (1986), y De Barbieri(1992) consideran que el género es una construcción cultural a partir de las diferencias anátomo-fisiológicas, de modo que los roles sociales asignados a hombres y mujeres pueden explicarse tomando en cuenta el modo en que se constituyen como formas de actuar y/o valorar en función a esos roles y no como resultado de las diferencias biológicas que distinguen los sexos. Así pues, la categoría género como base de una teoría sobre las 
relaciones de género permitió distinguir lo social de lo biológico superando la creencia de que las desigualdades en las relaciones de poder, relaciones económicas, domésticas, culturales, entre los individuos, eran determinadas por diferencias genéticas u hormonales, sino que por el contrario, estas desigualdades estaban muy relacionadas con el desarrollo de creencias, valoraciones y estilos que se estructuran en procesos de interacción social.

Pero esta postura llevó a algunas y algunos estudiosos del género a dar por válido lo social como determinante de distintos patrones de interacción de géneros, bajo el supuesto general de que "lo social determina patrones de desigualdad e inequidad en las relaciones de género", del cual se derivaron afirmaciones (o supuestos particulares) como las siguientes:

a. "los roles de género son determinados por los grupos sociales y, por lo tanto, se debe buscar eliminar la dominancia masculina" (Lomelin, 1995)

b. "que las mujeres hayan sido condicionadas a sentirse inferiores, secundarias, dóciles y complementarias del hombre y no como seres humanos iguales y capaces" (Randall, 1970)

c. "que la mujer es devaluada y oprimida en relación a la excesiva valoración o narcización del ideal masculino" (Jiménez, 1989);

d. otros supuestos que forman parte de las relaciones cotidianas como: "que determinados juguetes forman niños agresivos, machistas; mujeres sumisas, etc."

Tomando en cuenta que el género es una categoría que posibilita analizar las distintas maneras de relacionarse entre las personas en sociedad, también se hace necesario describir y explicar el cómo es que los factores, familiares, políticos, culturales e históricos, generan comportamientos mas o menos estereotipados, y el cómo es que esas variables participan de manera específica en el desarrollo de patrones de interacción social que pueden ser de igualdad o de desigualdad, de equidad o inequidad. Si se acepta el supuesto general de que lo social determina tipos determinados de comportamientos y valoraciones de los individuos, implicaría afirmar que los factores sociales son responsables de individuos estereotipados, machistas y segregadores. De este modo, los factores sociales estarían siendo entendidos como si fueran estímulos que causan conductas determinadas. 
Como ya se ha señalado en otra parte (Bazán, 1996), no basta con decir que los factores sociales influyen en la constitución de los roles de género igualitarios, segregados o estereotipados y se constituyen sobre la base de las diferencias biológicas (específicamente lo sexual), puesto que la dimensión social en el desarrollo psicológico no es algo que se superpone a las estructuras biológicas más, por el contrario, lo biológico implica, en el desarrollo psicológico, la condición necesaria para ciertas formas de comportamiento y como lo han señalado Carpio, Pacheco, Hernández y Flores (1995) es necesario considerar que el comportamiento se constituye a partir de la operación biológica del organismo, lo cual posibilita una serie de ajustes del individuo a distintas situaciones del ambiente con el que se relaciona funcionalmente. Debe quedar claro que ni lo social ni lo biológico deben entenderse como condiciones suficientes en la constitución de estilos de interacción de géneros ni de las creencias o valoraciones respecto a los roles masculinos o femeninos o el cómo vivir la sexualidad.

Una explicación muy acertada a cerca del género, es la de Ragúz (1995) quien considera que el género es una construcción psicológica y social, que es construido convencionalmente por una parte de la sociedad como un concepto relacional que hace mención a los diferentes contextos en los cuales se dan las relaciones humanas y en el que las mujeres y los hombres son distintos, no sólo por lo biológico, sino por la forma en que construye su noción y valoración del ser mujer o el ser varón. Esta forma de conceptualizar el género no lo reduce ni a lo psicológico, ni a lo sociológico, ni a lo biológico, pues es en lo social que se constituyen las valoraciones, creencias y actitudes hacia individuos de su propio sexo, comunidad, generación y a los de otros géneros, aún cuando se manejan términos que podrían cuestionarse pues hacen referencia a algún tipo de interiorización de roles, modelos y aprendizajes. Digo que este término podría ser cuestionable en función a que esta suposición podría conducirnos a la imperiosa necesidad de probar si los modelos y patrones sociales se interiorizan y, si se interiorizan, habría que detallar en qué lugar del mundo interno del hombre se almacenan estos modelos sociales.

El cómo es que se constituyen creencias, valoraciones y estilos de interacción de los individuos que se desarrollan en situaciones complejas de interacción social, ha inspirado a psicólogos con distintas orientaciones 
paradigmáticas a buscar explicar las formas en que se establecen los modos de interacción entre géneros. Por una parte, se ha estudiado la manera en que los factores de socialización, como la familia, la escuela, los medios de comunicación y las primeras relaciones en grupo social, influyen en los patrones estereotipados o de segregación de géneros. Un segundo grupo de estudios ha estado orientado a observar y/o analizar las preferencias y/ o creencias hacia actividades, juegos, y las emociones de las y los niños hacia sí mismos $u$ otras personas. Otros estudios se han realizado con la finalidad de explicar si los factores biológicos y genéticos influyen en el relacionamiento de los y las adolescentes en función al género (Mitchel, Backer y Jacklen, 1989).

A continuación se exponen algunos estudios alternativos a la explicación determinista de los roles y estilos de interacción de géneros sin que por ello signifique la propuesta de reducir la variable género a una categoría que implique solo lo psicológico. Por el contrario, estos estudios implican una forma de explicar el desarrollo de formas de interacción de los individuos bajo la categoría analítica del género.

Lynn, Wood y Little (1990) afirman que los estereotipos de género son adquiridos por los niños y niñas a través de formas complejas de asociación entre descriptores de género (lo masculino, lo femenino) y las relaciones de género (sus propias opiniones y creencias acerca de las características de personalidad, ocupación, apariencia física y comportamientos de otros individuos). Estos autores señalan que los niños y niñas adquieren roles estereotipados también haciendo inferencias en función a esos elementos o características. Sus investigaciones estaban dirigidas a probar si existe un aprendizaje selectivo de estereotipos y si la diferencia de edad en los juicios se basaba en el género. Para tal fin, los investigadores enseñaban a los sujetos de estudio, una tarjeta en la que estaba la figura de un niño o una nińa, le narraban una historia en el que el personaje de la figura era del sexo opuesto y estaba interesado por un juguete inconsistente para su sexo. El niño o nińa debería señalar a través de una escala de apariencias (que consistía de 3 figuras) qué tanto le gustan al nińo/ a de la tarjeta ciertos juguetes, a través de la presencia de tarjetas con el dibujo de varios niños/as y a partir de historias que incluían el interés de los personajes (masculino o femenino) hacia determinados juguetes. Los 
niños y niñas de 3 y 5 años debían contestar una escala de apariencias eligiendo una de las figuras que correspondía a una gran sonrisa (le gusta mucho), sonrisa moderada (le gusta regular y ceño fruncido (no le gusta nada). Los autores afirman que los niños y nińas realizan juicios basados sobre la información de su propio sexo, implicando asertividad en su propio sexo, y que cuanto mayor es la edad los juicios estarían en función a la información del sexo opuesto. Esto implicaría que conforme aumenta la edad $s$ hacen más evidentes las diferencias entre las características masculinas y femeninas.

Maccoby $(1990,1991)$ afirma que los roles de género se desarrollan en situaciones de interacción social en la que los niños y niñas adquieren diferentes estilos de interacción para adaptarse a los patrones estipulados para su propio sexo. Es decir, que las relaciones de género son formas de interacción social y que esas maneras de relacionarse (de interactuar) se constituyen como formas de desarrollo y no como causa de variables puramente biológicas o sociales. De esta manera, para Maccobby, las desigualdades en función al género se desarrollan desde la niñez a partir de patrones sociales con los cuales el individuo entra en contacto o relación. Estos contactos funcionales del individuo con su medio están convencionalmente establecidos. Los juegos por grupos segregados durante la primera infancia constituyen un fuerte ambiente de socialización en el que se experimentan distintas situaciones de interacción que son adaptadas a las del mismo sexo. Otros factores que, según Maccoby, contribuyen al desarrollo de patrones estereotipados, son las pautas de crianza, la influencia de los maestros de escuela, la familia, etc. Pero estos factores, por si mismos, no generan estilos estereotipados, sino que, por el contrario, estos factores dependen también de las formas especificas de actividades y conductas de los propios niños(as) y adolescentes. En otras palabras, los propios niños y niñas contribuyen a su desarrollo social ( $\mathrm{y}$ de género), como por ejemplo, son ellos y ellas quienes organizan reglas en una serie de juegos infantiles sin participación directa de adultos en dichas actividades. Es decir, que el efecto de las variables sociales en la individualidad en la niñez, se debe a que los niños y niñas también participan en asumir y transmitir los roles estereotipados solo para niños, sólo para niñas, o los roles aceptados para ambos. 
Karbon, Fabes, Carlo y Martín (1993) se propusieron identificar las creencias de los niños y las niñas preescolares acerca de la frecuencia e intensidad conque los hombres y mujeres experimentan enojo, tristeza y alegría y la forma cómo perciben los adultos y niños sus emociones. Es decir, que la percepción de la emoción de otras personas varía como una función del sexo y la edad del objeto/estímulo con el que interactúan las y los niños. Por ejemplo, las creencias de preescolares acerca de las diferencias sexuales en la tristeza están basadas sobre sus creencias en la capacidad de que la persona/estímulo pueda sentir tristeza. Por otra parte, las diferencias en relación al enojo percibido se dan sobre la base de las diferencias de edad y sexo en la frecuencia e intensidad. Por ejemplo, las personas adultas se enojarían mas que los niños y niñas y los hombres lo harían más que las mujeres. Las diferencias en la felicidad varían en función tanto del sexo, como el sexo del estímulo (la persona que funge como objeto-estímulo); por ejemplo, los niños creen que los hombres alcanzan mayor felicidad y las niñas creen que las mujeres son más felices.

Bayley y Zucker (1995) afirman que las preferencias hacia actividades con niños de su propio sexo se incrementan en los primeros ańos de la escuela. Estos autores realizaron una investigación con 16 niños y niñas de 4 años de edad para examinar si un conjunto de procesos intergrupales operan con respecto al género expresado como inclinaciones hacia niños y niñas del mismo sexo. Con tal fin, usaron películas para explorar la relación entre preferencias hacia el propio sexo y otros fenómenos intergrupales. Un aspecto importante de este estudio fue el hecho de que los individuos modelo (objetos/estímulo) se referían a un niño o a una niña extraña quienes participaban en distintas situaciones de interacción con adultos, considerado como contexto de interacción neutra en relación al género; es decir, que las escenas de las películas indicaban actividades no estereotipadas. En este estudio se observó que los objetos de estímulo, tanto masculinos como femeninos, recibieron puntajes más altos de preferencia por niños y nińas del propio sexo que por los del sexo opuesto. Estos hallazgos reflejan que la actitud acerca de otros niños y niñas que le son extrañas no se basa solamente en los estilos de interaccion que presentan en la actualidad cuando interactúan con niños de su edad, sino que ésto está en función a su historia de interacción con otras personas, sean estos hombres o mujeres, niños o nińas. 


\section{Estilos de interacción de géneros}

Un aspecto muy importante del quehacer psicológico en el análisis de las relaciones de género debe orientarse a explicar la manera en que los diversos factores se intercalan entre si e influyen de distintas formas en la constitución de un estilo o rol de género como tendencias interactivas relativamente invariantes. Estos factores están estrechamente vinculados al desarrollo de actitudes, valoraciones y competencias como formas de ejecución de ciertas actividades consideradas sólo para hombres o sólo para mujeres, lo cual ocurre en situaciones particulares de interacción social y está en función a las distintas formas de organización de los contextos en los cuales se dan dichas relaciones. Es decir, que dentro del supuesto de que la Psicología tiene por objeto el formular enunciados que describan las relaciones generales entre hechos particulares de cierta clase y las condiciones en que dichas relaciones tienen lugar, es necesario enfatizar que las diferencias singulares del comportamiento implican diferencias características de cada individuo como diferencias que lo tipifican como un individuo que se comporta en contextos específicos de interacción. Adicionalmente, es oportuno señalar que esas distintas maneras en que los individuos se relacionan con objetos y eventos en determinados contextos se constituyen históricamente a través de interacciones específicas en las que las características funcionales del ambiente son determinadas fundamentalmente por la práctica convencional de los grupos humanos. Estos participan en el proceso de socialización del comportamiento de los individuos limitando, por una parte, los criterios de logro que los individuos deben alcanzar cuando se comportan en los distintos periodos de desarrollo y de vida en sociedad y, por otra parte, estableciendo los criterios de valoración que regulan tales comportamientos, como por ejemplo, lo adecuado o inadecuado, lo normal o lo anormal, lo deseable o lo indeseable, entre otros. En este sentido, la categoría género debe permitir analizar y describir los procesos a través de los cuales se establecen una serie de actitudes, preferencias y estilos de interacción en ciertos contextos, los cuales establecen qué actividades son propias de un determinado sexo, edad, clase social, etc., y le asignan valores distintos a cada actividad. Por ejemplo, expresiones como: «los niños no lloran», "las niñas no agreden", "la rebeldía es propia de los jóvenes", "no te juntes con los hijos de los negros o indios", regulan la constitución de tendencias interactivas, pero 
no determinan que las niñas puedan ser, más adelante, mujeres sumisas, incapaces de levantarle la voz a su marido quien, por "naturaleza", es fuerte y a quien socialmente se le permite agredir a la mujer.

En esta perspectiva, se hace importantísimo describir el proceso por el cual lo psicológico se constituye de manera distinta a lo social. En otras palabras, el identificar cómo es que la especificidad histórica del individuo se constituye como modos particulares de participar en procesos de interacción social en las que lo social implica la constitución en lo colectivo de características comunes que diferencian los comportamientos de hombres y mujeres, niños y ancianos, blancos y negros. A juicio del autor de este trabajo, la taxonomía de Ribes y López (1985) acerca de la jerarquización del comportamiento en niveles de organización y complejidad funcional, y la propuesta para el análisis del desarrollo de la individualidad de Ribes (1990a, 1990b) ofrecen importantes criterios que pueden tomarse en cuenta para elaborar categorías de análisis de estilos de interacción de géneros y para describir patrones de desarrollo de estilos y de creencias en las relaciones de género.

Del análisis anterior en este trabajo se postula que el tema de las relaciones de género significa un interesante objeto de estudio para la Psicología, puesto que implica hechos particulares relativos al comportamiento de los individuos en situaciones variadas de interacción. En tal virtud, se propone que el quehacer psicológico en el tema de relaciones de género debe tomar en cuenta los siguientes aspectos:

1. Asumir una lógica conceptual que ampare el análisis de la dimensión psicológica de las relaciones de género sin tomar criterios explicativos de otras disciplinas aún cuando el género hace mención a distintas dimensiones que lo haría objeto de estudio de diversas disciplinas.

2. Derivado de esta propuesta conceptual, construir una serie de taxonomías o categorías para describir y explicar el cómo se constituyen los distintos modos de relación de géneros.

3. El ejercicio del análisis conceptual y las propuestas para describir el cómo se desarrollan las relaciones de género debe insertarse dentro de una perspectiva y una práctica interdisciplinaria sin tratar de reducir a lo psicológico las otras dimensiones a las que también hace referencia la categoría género, pero respetando el análisis de los aspectos psico- 
lógicos desde criterios establecidos para explicar lo psicológico. Esto posibilitaría al psicólogo interesado en este tema, asumir una perspectiva más amplia acerca de cómo es que esos mecanismos o factores socializantes disponen las condiciones para la adquisición, mantenimiento y/o modificación de los estilos y roles de género que, a su vez, están expuestos a un constante desarrollo en función a la variación de las condiciones en las auales se establecen las interacciones sociales.

De acuerdo a estos criterios, y como ya se ha advertido, lo social no puede entenderse sólo como el factor que genera tal o cual tipo de comportamiento de los individuos sino que, más bien, lo social debe entenderse como la forma en que el individuo desarrolla distintas maneras de interacción de acuerdo a las distintas formas en que se organiza su contacto funcional con una serie de factores en diferentes situaciones específicas. Dado esto, las diferencias en el comportamiento de los individuos (hombres o mujeres) de diferentes edades, diferentes condiciones económicas, de distintas razas, etc., son diferencias resultantes de aprendizajes de formas de comportarse y de prácticas valorativas de los roles masculino y femenino y de las formas de comportamiento compartidos por los miembros de una clase social, generación, sexo y, también, de sus propias creencias y valoraciones. Así, las similitudes del comportamiento de los miembros de un género se conforman a través de procesos similares o de experiencias de vida que son compartidas en los contextos de interacción, que incluyen el cómo se vive y el cómo se valora el ser niño o niña, hombre o mujer, el pertenecer a una clase económica pudiente o pobre, el cómo se experimenta la adolescencia o la senectud, etc. En este sentido, no se debe sobredimensionar ni la similitud intragenérica ni las diferencias intergenéricas, puesto que el desarrollo psicológico implica procesos particulares de desarrollo para cada individuo, de modo que estos individuos se comportan y muestran habilidades, destrezas y valoraciones de manera muy distinta.

\section{Referencias}

Bazán, A. (1996). La dimensión psicológica del género. Ponencia presentada en: Mujer, Género y Desarrollo; II Encuentro Internacional de Investigaciones y Estudios de Género. Michoacán, 56 y 7 de noviembre. 
Bailey, J. y Zucker, K. (1995). Childhood sex-typed behavior and sexual orientation: A conceptual analysis and quantitative review. Developmental Psychology, 31 (1) 43 - 55.99

Carpio, C. Pacheco, V. Hernández, R. Y Flores, C. (1995). Creencias, criterios y desarrollo psicológico. Acta Comportamentalia, 3 (1), 8998.

De Barbieri, T. (1992). Sobre la categoría género. Una introducción teórica-metodológica. Isis Internacional: Ediciones de las Mujeres, 17, 11-128.

Jiménez, D. M. (1989). La constitución del ser mujer desde la vida cotidiana. En: Jiménez, D. M. (Ed), Estudios de género y feminismo. México: Fontamara.

Karbon, M. Fabes, R. Carlo, G. y Martin, C. (1993). Preschooler's beliefs about sex and age differences in emotionality. Sex Roles, 27 (7/8), 377390.

Lamas, M. (1986). La antropología feminista y la categoría "género". Nueva Antropologia, 8 (30), 173-198.

Lomelin, M. (1995). El cuerpo y la sexualidad para la construcción en la noción de género. Ponencia presentada en el XX Congreso de la Asociación Latinoamericana de Sociología: Perspectivas de su Reconstrucción. México.

Lynn, C. Wood, C. \& Little, J. (1990). The development of gender stereotype components. Child Development, 61, 1891-1904.

Maccoby, E. (1990). Gender relationships: A developmental accounts. American Psychologist, 45 (4), 513-520.

Maccoby, E. (1991). Gender y relationships: A response. American Psychologist; 46 (5), 538-539.

Mitchel, J. Backer, L. \& Jacklen, N. (1989). Masculinity and feminity in twin children: Genetic and enviromental factors. Child Development $60,1475-1485$.

Ragúz, M. (1995). Construcciones sociales y psicológicas de mujer, hombre, femineidad, masculinidad y género, en diversos grupos poblacionales. Lima: Pontificia Universidad Católica del Perú/Lluvia Editores.

Randall, M. (1970). Las mujeres. México: Siglo XXI.

Ribes, E. (1990a). El problema de las diferencias individuales: Un análisis conceptual de la personalidad. En: E. Ribes, E. (Ed), Psicologia General. México: Trillas. 
Ribes, E. (1990b). La individualidad como problema psicológico: El estudio de la personalidad. Revista Mexicana de Análisis de la Conducta $16(3), 7-24$.

Ribes, E. y López, F. (1985). Teoría de la conducta: Un análisis de campo y paramétrico. México: Trillas .

Rubin, G. (1984). El tráfico de mujeres: Notas sobre la "economía política" del sexo. Nueva Antropologia 8 (30), 95-145. 